\title{
An Efficient Video Content Delivery Strategy for Radio Access Network Environments
}

\author{
Po-Jen Chuang and Hang-Li Chen \\ Department of Electrical Engineering \\ Tamkang University \\ Tamsui, New Taipei City, Taiwan 25137, R. O. C. \\ E-mail:pjchuang@ee.tku.edu.tw
}

\begin{abstract}
When a radio access network (RAN) environment cannot handle the tremendous mobile data traffic, users may experience degraded or declined services. To help process the large number of user video requests in RANs, a content delivery network (CDN) architecture has been recently introduced, which establishes a number of cache servers outside the source server for users to get the needed data from the nearest cache server. To upgrade the performance of a wireless CDN, this paper presents a new caching strategy based on analytical results of real user video request traces and specific caching considerations for videos with different popularity degrees. The new strategy first caches videos with high popularity to all helpers and then caches those with low popularity to the remaining helper storage to enhance the overall request hits. Performance evaluation exhibits that, when compared with other caching strategies, our new strategy is able to yield better request hits with low complexity and average delay time.
\end{abstract}

Keywords: Content delivery networks (CDNs), radio access networks (RANs), caching strategies, video request hits, complexity, delay, experimental evaluation

\section{Introduction}

According to the visual networking index [1] released by Cisco, the average monthly global mobile data traffic was $1.5 \mathrm{~EB}$ in 2013 and will grow up to $15.9 \mathrm{~EB}$ in 2018 with about $70 \%$ of it coming from video traffic data. By that time, the radio access network (RAN) environments (as shown in Figure 1) will have very serious problems absorbing the estimated huge growth of mobile data traffic. In fact, the trouble may emerge earlier in 2017 as Strategy Analytics has forecasted. When it happens, users may experience degraded or even declined services because the base station in RANs will have difficulties handling the large number of user video requests [2]. If viewing the base station as a server, we can thus describe the foreseeable problem: A single server may fail to manage too many user requests and will then respond to these requests with extra delay time. To solve the incoming problem, some researchers have recently introduced the content delivery network (CDN) architecture [3-5] to assist the practice of RANs.

In the $\mathrm{CDN}$ architecture, a number of cache servers are established outside the source server. The source server then caches its contents to the cache servers so that users, when filing requests, can get the needed data from the nearest cache server. Such a practice can increase the delivery speed and reduce the burden on the source server. The performance of CDN architectures is subject to a proper caching strategy by which the source server can decide how to set up desirable cache servers and to cache what contents to them. A number of caching strategies have been proposed for the CDN architecture, including [615]. For instance, to facilitate the performance of a wireless CDN architecture, the Greedy strategy [6-7] creates several cache nodes (the so-called helpers) in a single cell. It faces a major problem: When the video volume grows much bigger than the cache 
storage of helpers, the strategy can only cache the videos with high popularity. For the considerable number of videos with low popularity, it will turn over very limited user request hits.

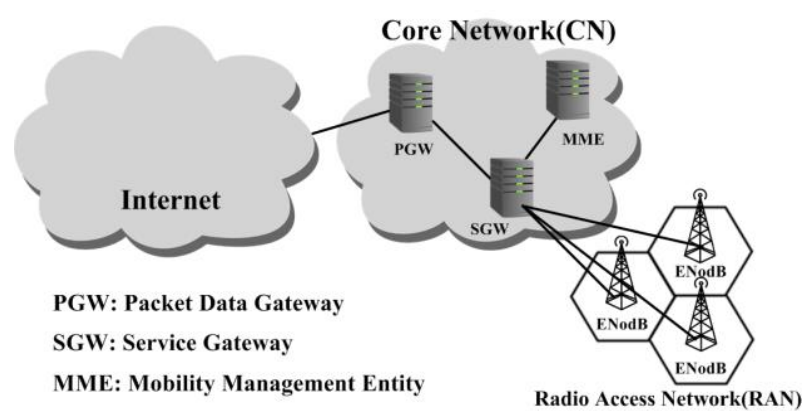

Figure 1. An Illustration of the Radio Access Network (RAN)

The main goal of this investigation is to enhance the practice of RANs by building a new caching strategy over the wireless CDN architecture. Different from the Greedy strategy, our new caching strategy analyzes the real user video request traces and, based on the results, adopts different approaches to handle videos with different degrees of popularity. Our basic idea is to cache videos with high popularity to all helpers first and then cache those with low popularity to the remaining storage (if the total storage of helpers is not full). By doing so, we can effectively lift up the request hits of those less popular but numerous videos.

Extensive simulation runs are conducted to evaluate the performance of our cache strategy and other CDN cache strategies, including the Greedy [6, 7], Popular [7], and Fuzzy Decision [8] strategies. The obtained results show that, when the cache storage of helpers goes below $50 \%$ of the total videos (i.e., with limited helper cache storages), the Greedy strategy attains only few hits for the big number of less popular videos. The Popular strategy is shown to yield much lower request hits because it caches only those highly popular videos, and the Fuzzy Decision strategy misses even the requests of highly popular videos because it does not consider video popularity at all. By contrast, our new strategy performs following the real user request traces and therefore is able to achieve higher request hits with lower complexity. We also use the widely applied LTE-sim [1619] to simulate these target strategies and obtain the average video download delay time per user, with the advantage to our strategy as well.

\section{The Proposed Strategy}

When caching a video, the Greedy strategy must ensure that each user can find the video in a nearby helper. But, it is difficult to predict accurately which user will make a request in which position and, meanwhile, wrong user location predictions will bring up huge impact. If users are evenly distributed, the strategy needs to cache every video to most of the helpers. On the other hand, if the video volume largely exceeds the total helper storage, helpers can cache only limited videos. In such a situation, the Greedy strategy which has attempted to handle most user requests by nearby helpers will have to leave a significant amount of requests untended. To solve the problem, we build a new caching strategy based on the analytical observations of real user request traces to increase the probability of having helpers handle user requests, i.e., to increase the user request hits.

To facilitate our investigation, we examine the traces of the YouTube requests extracted from the wired campus network, between the second half of 2007 and the first 
half of 2008, at the University of Massachusetts at Amherst [20-21]. Table 1 gives one example of these request traces.

Table 1. The Trace of a YouTube Request

\begin{tabular}{|l|l|l|l|l|l|}
\hline Timestamp & $\begin{array}{l}\text { YouTube } \\
\text { server IP }\end{array}$ & Client IP & Request & Video ID & Content server IP \\
\hline 1189828805.208862 & 63.22 .65 .73 & 140.8 .48 .66 & GETVIDEO & 1ML9dik8QNw & 158.102 .125 .12 \\
\hline
\end{tabular}

We take the number of requests for each video as its popularity and observe that, each day, the average user requests for videos with higher popularity (i.e., with more requests) stands about $30 \%$ of the total requests. We also observe that, when removing these $30 \%$ more popular videos, the remaining $70 \%$ videos exhibit little popularity differences. To facilitate our later discussions, we now define those more popular videos as popular videos and the rest as non-popular videos.

Assume that there are $N$ videos and the popularity (i.e., the number of requests) of video $i$ is $P i$. We first accumulate the popularity of the $N$ videos in set $S$, in descending order, i.e., $P i-1>=P i$.

$$
S=\left\{P_{1}, P_{2}, \ldots, P_{N}\right\}
$$

Asmentioned previously, the requests forpopular videos stand about $30 \%$ of the total video requests. We can thus definethe total requestsfor popular videos (denoted as Ppopular) as

$$
\begin{gathered}
P_{\text {total }}=\sum_{i=1}^{N} P_{i} \\
P_{\text {popular }}=P_{\text {total }} \times 30 \%
\end{gathered}
$$

With the above definitions, we can start our first phaseof operation: caching all popular videos to all of the helpers. Figure 2 gives the details of the caching flow.

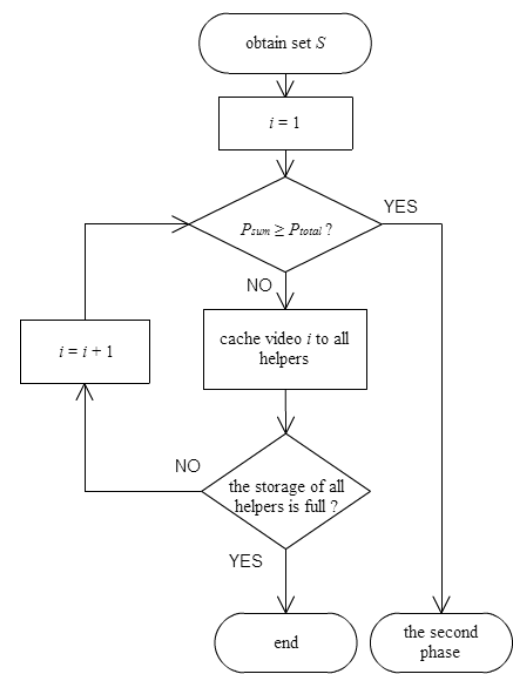

\section{Figure 2. The Flow Chart of the First Phase: Caching Popular Videos to All Helpers}

In Figure 2, $P_{\text {sum }}$ (initially 0 ) indicates the total popularity of the cached videos. When $P_{\text {sum }}$ remains below $P_{\text {popular }}$, cache the next popular video to all helpers; repeat the same operation until the caching storage of all helpers is full or $P_{\text {sum }}$ equals/exceeds $P_{\text {popular }}$.

If the cache storage of helpers is not used up after the first phase, we will start the second phase of operation. Assuming that we have cached $H$ videos out of the total 
$N$ videos, $H<N$, to all helpers in the first phase, we now have the following $S_{\text {non-popular }}$ (the N-H non-popular videos) set

$$
S_{\text {non-popular }}=\left\{P_{H+1}, P_{H+2}, \ldots, P_{N-1}, P_{N}\right\}
$$

As the analytical results of the YouTube requests exhibit little popularity differences for non-popular videos, we thus evenly distribute these non-popular videos to the helpers without considering the popularity. If we assume all videos are with the same size and each helper has the cache storage for $K$ videos, we will attain the total cache storage of $M$ helpers as

$$
\text { Storage }_{\text {total }}=M \times K \text { videos }
$$

Excluding the $H$ videos which have been cached in the first phase, we now have the following remaining cache storage

$$
\text { Storage }_{\text {surplus }}=M \times(K-H) \text { videos }
$$

That is, we can distribute the remaining non-popular videos to the helpers

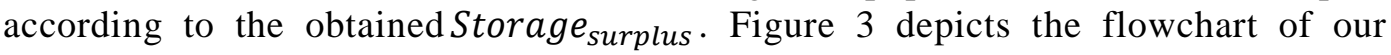
second phase of operation.

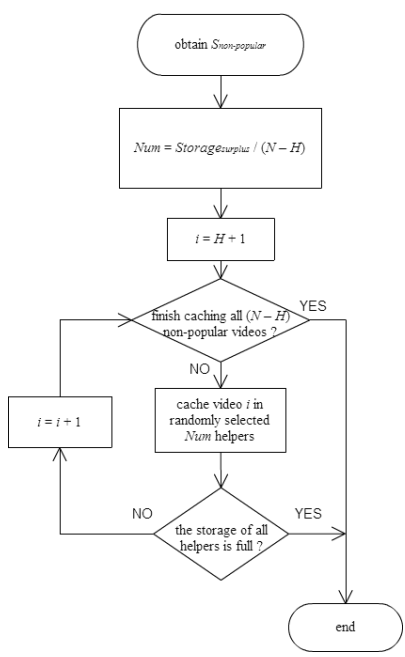

\section{Figure 3. The Flow Chart of the Second Phase: Caching Non-Popular Videos}

In Figure 3, Num indicates the quotient of the remaining cache storage divided by the total number of non-popular videos; each non-popular video hence can be cached to Num helpers. For a non-popular video $i, i=H+1, H+2, \ldots, N-$ $1, N$.Whena video (starting from $H+1$ ) is cached to the Num helpers randomly, the remaining cache storage will be decreased until the $N-H$ videos are all cached or Storage surplus $^{\text {is used up. }}$

To sum up, the proposed caching strategy adopts different approaches to handle popular and non-popular videos. In the first phase, it caches the popular videos -one by one, in the order of popularity -- to all helpers until using up the helper storage or the popular videos. This is a different approach in contrast to the Greedy strategy which starts by caching each video to most of the helpers and consequently occupies too much helper storage in the beginning. By caching popular videos, instead of all videos, in the beginning, we can practically save storage and ensure helpers to come up with proper request hits. Such a design can also avoid the loophole of the Fuzzy Decision strategy. (Note that helpers in the Fuzzy Decision strategy may fail to handle quite a few popular requests as it will randomly cache 
videos several times and select the best result without considering popularity degrees.)

After the practice of the first phase, if the helpers still have cache storage, our strategy will start the second phase of operation. As the above trace analysis of the YouTube requests shows little popularity difference for non-popular videos, our strategy evenly distributes these non-popular videos without considering the popularity to the helpers in the second phase. Our key consideration in this phase is to cache possibly more non-popular videos to the helpers. By doing so, we can further enhance the request hits, given that most of the video requests are for nonpopular videos (e.g., 70\% in the above You Tube case). Unlike the Greedy strategy which caches very few non-popular videos or the Popular strategy which caches only popular videos, our strategy can cache significantly more non-popular videos to the helper (so as to increase the request hits), thanks to its second-phase practice. Our strategy is relatively simple because it deals with the popularity degrees of videos only, not involving user location predictions as the Greedy strategy. For better understanding we list critical features of different caching strategies in Table 2 .

Table 2.Critical Features of Different Caching Strategies

\begin{tabular}{|c|c|c|c|c|}
\hline & Greedy & Popular & $\begin{array}{c}\text { Fuzzy } \\
\text { Decision }\end{array}$ & Ours \\
\hline Environment & wireless & wireless & wired & wireless \\
\hline $\begin{array}{c}\text { Popularity } \\
\text { information }\end{array}$ & required & required & not required & required \\
\hline Users' positions & required & not required & required & not required \\
\hline
\end{tabular}

\section{Experimental Evaluation}

\subsection{The Simulation Environment}

To evaluate the performance of our new caching strategy (ours) and other related strategies, including the Greedy, Popular and Fuzzy Decision strategies, we carry out extensive simulation runs in a created single cell of the RAN environment with a number of helpers as Figure 4 depicts. We pick up user request traces by randomly selecting from the You tube requests (specified in the previous section) which had been filed during the four hottest hours (i.e., hours with the most incoming requests)on January 29, 2008:a total of 5252 requests filed for 3888 different videos. Each requested video is assumedly with resolution $=640 \times 360$, video length $=$ three minutes and video size $=30 \mathrm{MB}$. To attain fair simulation, we suppose the popularity of each video is known in advance and meanwhile follow the environment settings of the Greedy strategy in[7] --with cell radius $=400$ meters and a random set of helpers in the cell $=32$. The performance of the four target strategies is collected and compared in terms of video request hits and complexity. (Note that our simulation involves LTE- sim [16] to obtain the average delay each user takes to download a video.) 


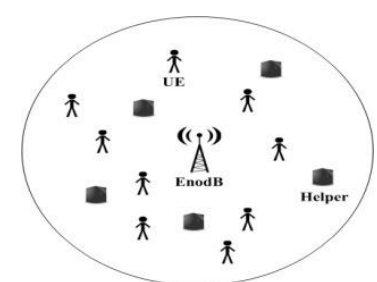

Figure 4.The Simulation Environment

We build the helper's model like the Greedy strategy. The connections between the helpers and users are Wi-Fi-like D2D (device-to-device) communications [7], and the transmission speed between the helpers and users is assumed by using $802.11 \mathrm{n}$ [22]. Table3 gives related parameters for the helper's model.

Table 3. The Helper's Model

\begin{tabular}{|c|c|}
\hline Parameter & value \\
\hline helper range & $100 \mathrm{~m}$ \\
\hline data rate $(\mathrm{Mbps})$ & $\mathrm{R}=3+(4 / 100) *(100-\mathrm{d})$ \\
\hline
\end{tabular}

Table 4 gives related parameters of the base station and channel models. We use the LTE-sim with the following parameters to simulate the environment of 3GPPSpecificationsRelease 8.

Table4. The Base Station and Channel Models

\begin{tabular}{|c|c|}
\hline Parameter & value \\
\hline bandwidth & $15 \mathrm{MHz}$ \\
\hline subcarrier spacing & $15 \mathrm{kHz}$ \\
\hline number of subcarriers per OFDM symbol & 12 \\
\hline slot duration & $0.5 \mathrm{~ms}$ \\
\hline scheduling time (1 TTI) & $1 \mathrm{~ms}$ \\
\hline schedulers & proportional fair(PF) \\
\hline Path Loss Model For Urban Environment & L $=128.1+37.6 * \log _{10} \mathrm{~d}$ \\
\hline
\end{tabular}

\subsection{Simulation Results}

\subsubsection{Video Request Hits vs. the Helper Storage}

To see how helper storage relates to the video request hits, we carry out simulation runs with different volumes of helper storage ranging from 30GBs to $90 \mathrm{GBs}$. Figure 5 depicts the numbers of video request hits versus the helper storage (in GBs) for different strategies. As we can see, when the helper storage is small, the Popular strategy yields fewer video request hits because it caches only the popular videos. For the Fuzzy Decision strategy which considers no popularity degrees of the videos, increasing the helper storage does not distinctly increase the request hits. It yields better request hits than Popular only at smaller cache storage (likely because of caching more different videos). Our strategy depicts the best hits between cache storage 30 60GB because we heed not only the hits for popular videos but also that for the non-popular videos. The Greedy strategy generates more 
desirable video hits than our strategy between cache storage 60 90GB, mainly because it can follow user location predictions to make sure videos be cached in the neighboring helpers when there is sufficient helper storage.

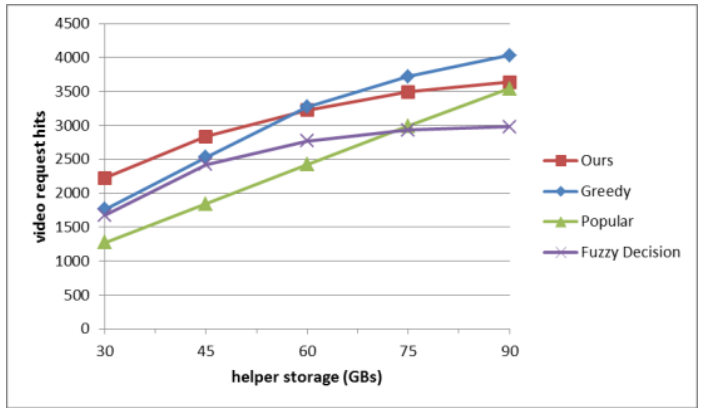

Figure 5. Video request hits vs. the helper storage (GBs).

\section{Figure 5. Video Request Hits vs. the Helper Storage (GBs)}

Note that the 5252 video requests covered in our simulation are the accumulated requests in four consecutive hours, each respectively having 824, 1457, 1732 and 1239 requests. Figure 6 exhibits the accumulated request hits of the four hours with helper storage $=30 \mathrm{~GB}$ (where $\mathrm{n}$ in the $\mathrm{x}$-axis indicates the first $\mathrm{n}$ hours). The results show that, for the Popular strategy, users make requests for popular videos mainly in the first two hours (which have more focused accumulated request hits). In the last two hours, we can see more sporadic non-popular videos have been requested. Our new strategy, by contrast, enables the request hits to grow continuously even during the last two hours. This happens because we endeavor to raise the request hits for both the popular and non-popular videos. The Greedy strategy displays quite leveling performance during the final hour, a result of yielding lower hits for non-popular video requests.

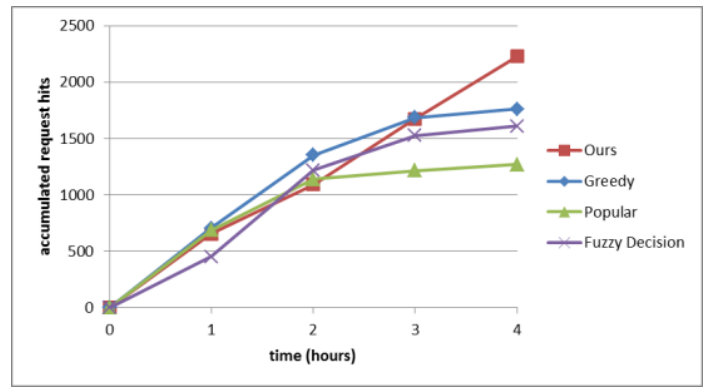

Figure 6. Accumulated request hits vs. time (hrs) (30GB helper)

Figure 7 depicts the accumulated request hits of the same four hours, with 60GB helper storage which is able to cache about $50 \%$ of the total videos. With 60GB helper storage, the Greedy strategy can upgrade its request hits to catch up with our strategy, because it has bigger helper storage to cache more videos and is with the assistance of user positions. As for the Popular strategy, we can see it attains better request hits for the first two hours because users make more requests on popular videos during that period of time. The result also reveals that the request hits for the Fuzzy Decision strategy display the same developing trend as that for our strategy, but the Fuzzy Decision strategy constantly yields lower request hits than our strategy because it does not take the popularity of videos into account. 


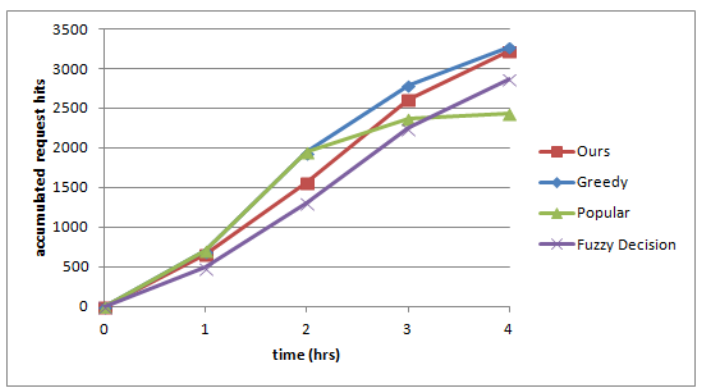

Figure 7. Accumulated request hits vs. time (hrs) (60GB helper).

Figure 8exhibits the accumulated request hits of the above four hours with 90GB helper storage which can cache about $80 \%$ of the total videos. When the helper storage grows to $90 \mathrm{~GB}$, each helper will be able to cache most of the videos. In such a situation, the Greedy strategy turns over higher request hits than our strategy with the aid of user positions. That is, when the helper storage grows bigger, caching more kinds of videos (popular or non-popular) turns less significant. It helps explain why, in Figure 8, our strategy trails behind the Greedy strategy in request hits. However, as mobile data traffic will grow tremendously in the future (mentioned in the beginning of Section 1), it will be impossible for a helper to cache over $50 \%$ of the total videos by that time because, to do so, a helper will need extremely massive caching storage which may group to hundreds of GBs. With hundreds of GBs storage, we can foresee escalating transmission delay due to a lot of factors, including cache update. In view of this prospect, caching strategies should be designed for practice in reasonable environments, i.e., when helpers are with more reasonable caching capability.

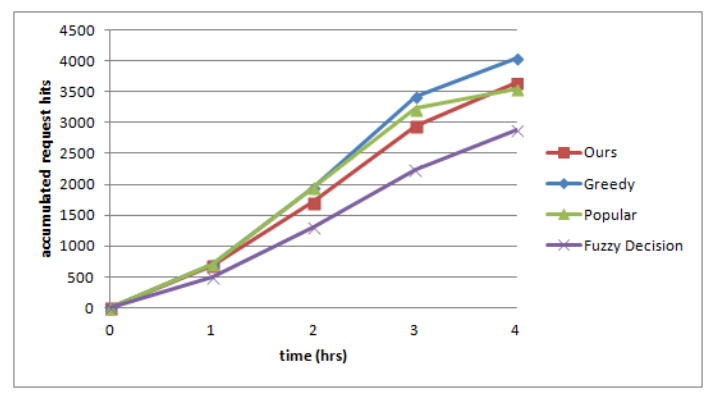

Figure 8. Accumulated Request Hits vs. Time (hrs) (90GB Helper)

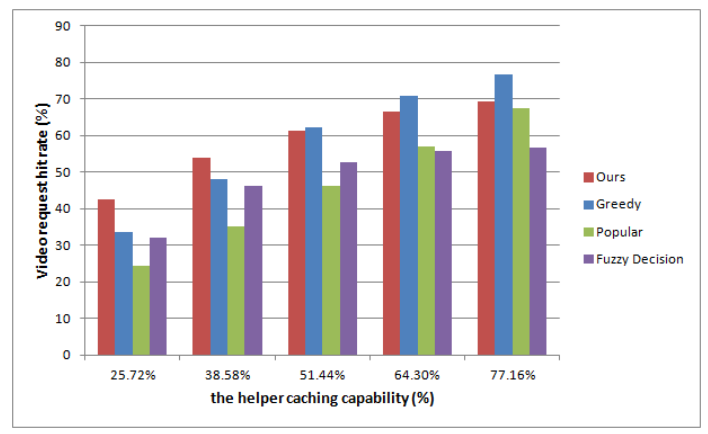

Figure 9. Video Request Hit Rates vs. the Helper Caching Capability 


\subsubsection{Video Request Hits vs. the Helper Caching Capability}

Figure 9 gives that result of video request hits versus the helper caching capability. It further illustrates the advantage of our strategy, that is, when helpers are with reasonable caching capability, our special design out of the popularity degrees of videos can ensure more desirable performance in request hits. Note that the helper caching capability in Figure 9 indicates the percentage of total videos each helper can cache and the request hit rate indicates the number of request hits/the number of total requests. As we can see, our strategy yields higher request hit rates when the helper caching capability is under about $50 \%$-- which is the more reasonable case.

\subsubsection{Computational Complexity}

Figure 10 gives computational complexity denoted by the number of lookups in the helper storage, such as the lookup for a requested video or the lookup to see if the helper storage is full upon caching a video. The result shows that the complexity of the Popular strategy equals the total number of videos to be cached by the helpers, and that our strategy (which randomly caches the non-popular videos) produces slightly higher complexity than the Popular strategy. It also shows that the Fuzzy Decision strategy produces higher complexity than Popular and our strategy because it needs 10 runs of random caching before reaching caching decisions. Among all strategies, the Greedy strategy takes the most complexity because it needs to search all user locations in order to decide on the appropriate helper for caching each of the videos.

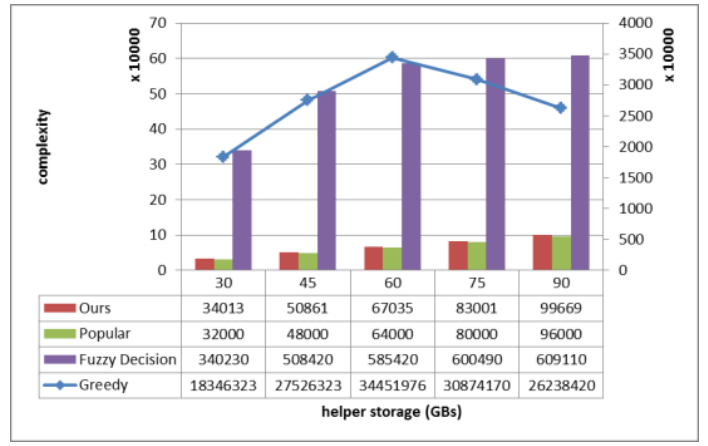

Figure 10. Complexity vs. Helper Storage

\subsubsection{Average Delay Time}

In the simulation, we also use LTE-sim and the helper model to simulate the average delay time for a user to download a video by the strategies. The average delay time will be attained by the following steps:

(1) Use LTE-sim to simulate delay time for a user to download one video from the base station.

(2) Use the helper model to calculate the delay time for a user to download one video from the helper.

(3) Average the sum of the above two delay times to get the estimated average delay time.

The result of the average delay versus the helper storage is plotted in Figure 11. Note that the Baseline strategy in this simulation assumes all requests are served by the base station, i.e., it uses no wireless CDN architectures. As the result illustrates, each strategy-except Baseline can shorten the delay time in a trend corresponding to the results of 
request hits in Figure 5. In fact, the undesirable result of the Baseline strategy helps prove the feasibility of using wireless CDN architectures to deal with the problems which will confront the RAN sin the near future.

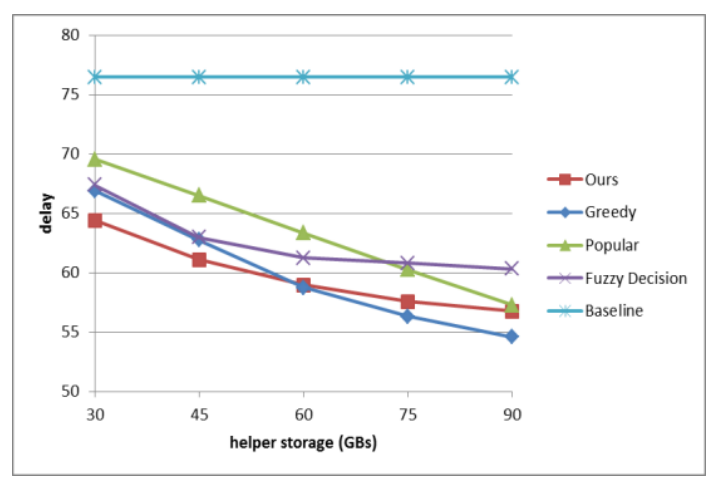

\section{Figure 11.The Average Delay vs. the Helper Storage}

\section{Conclusions}

Considering that the radio access network (RAN) environments will face very serious challenges in managing colossal growth of mobile data traffic in the near future, a content delivery network (CDN) architecture has been proposed to assist the practice of RANs. The CDN architecture sets up a number of cache servers outside the source server which then caches its contents to them. When a user files requests, it can get the needed data from the nearest cache server to increase the delivery speed and reduce the burden on the source server. A proper caching strategy is therefore critical to the performance of CDN architectures as it helps decide how to set up desirable cache servers or to cache what contents to them. To facilitate the performance of a wireless CDN architecture, this paper introduces a new caching strategy based on the analytical results of real user video request traces. The new strategy adopts different caching approaches to handle videos with different degrees of popularity. It first caches videos with high popularity to all helpers and then those with low popularity to the remaining storage. The special design helps increase the request hits of those less popular but large-numbered videos and upgrade the overall performance of a CDN. Experimental evaluation shows that, in contrast to related strategies, our new caching strategy is able to achieve higher request hits with significantly lower complexity and average delay time.

\section{References}

[1] Cisco Visual Networking Index, Forecast and Methodology, Available: http://www.cisco.com/en/US/solutions/collateral/ns341/ns525/ns537/ns705/ns827/white_paper_c11481360_ns827_Networking_Solutions_White_Paper.html, (2013-2018).

[2] S. Rudd, "Global Backhaul Investment Gap of \$9.2 Billion could increase Customer Churn." Available: http://www.strategyanalytics.com/default.aspx?mod=pressreleaseviewer\&a0=5332.Lazar and W. Terrill, "Exploring content delivering networking," in IT Professional, vol. 3, no. 4, (2001) August, pp. 47-49.

[3] G. Pallis and A. Vakali, "Insight and Perspectives for Content Delivery Networks," in Communications of the ACM - Personal information management, vol. 49, no. 1, (2006) January, pp. 101-106.

[4] M. Pathan and R. Buyy, "A taxonomy of CDNs, Content Delivery Networks," in Germany: SpringerVerlag, (2008).

[5] N. Golrezaei, K. Shanmugam, A. Dimakis, A. Molisch and G. Caire, "Fem to caching: Wireless video content delivery through distributed caching helpers", Proc. 2012 IEEE INFOCOM, (2012) March, pp. $1107-1115$. 
[6] N. Golrezaei, A. G. Dimakis, A. F. Molisch and G. Caire, "Fem to caching and device-to-device collaboration: A new architecture for wireless video distribution", IEEE Communications Magazine, vol. 51, no. 4, (2013), pp. 142-149.

[7] J. B. Chen, "Efficient content placement on multimedia CDN using fuzzy decision algorithm", Applied Mathematics \& Information Sciences, vol. 6, no. 2, (2012) April, pp. 471-477.

[8] L. Qiu, V. Padmanabhan and G. Voelker, "On the placement of web server replicas," in INFOCOM 2001. Twentieth Annual Joint Conference of the IEEE, (2001) April, pp. 1587-1596.

[9] S. Sivasubramanian, M. Szymaniak, G. Pierre and M. van Steen, "Replication for web hosting systems," in ACM Computing Surveys, vol. 36, no. 3, (2004) September, pp. 291-334.

[10] B. R. Tan, and L. Massouli e, "Optimal content placement for peer-to-peer video-on-demand systems," in IEEE/ACM Transactions on Networking, vol. 2, no. 2, (2013) April, pp. 566-579.

[11] S. Borst, V. Gupta and A. Walid, "Distributed caching algorithms for content distribution networks," in Information communications, 29th conference, (2010) March, pp. 1478-1486.

[12] H. Huang, P. Xia, S. G. Chan, G. Shi and H. Zhang, "Joint optimization of content replication and server selection for video-on-demand", IEEE International Conference on Communications, (2012) June, pp. 2065-2069.

[13] W. Jiang, S. Ioannidis, L. Massoulíe and F. Picconi, "Orchestrating massively distributed CDNs," in ACM Co NEXT, (2012).

[14] J. P. Muñoz-Gea, J. Malgosa-Sanahuja and P. Manzanares-Lopez, "Optimizing content placement in a peer-assisted VoD architecture", Peer-to-Peer Networking and Applications, vol. 6, no. 3, (2013) September, pp. 340-360.

[15] G. Piro, L. Grieco, G. Boggia, F. Capozzi and P. Camarda, "Simulating LTE cellular systems: An opensource framework", IEEE Transactions on Vehicular Technology, vol. 60, no. 2, (2011) Febuary., pp. 498-513.

[16] C. Bouras, N. Kanakis, V. Kokkinos and A. Papazois, "Application layer forward error correction for multicast streaming over LTE networks", International Journal of Communication Systems, (2012) February.

[17] C. Xu, F. Zhao, J. Guan, H. Zhang and G. M. Muntean, "QoE-driven user-centric VoD services in urban multi-homed P2P-based vehicular networks", IEEE Transactions on Vehicular Technology, vol. 62, no. 5, (2012) November, pp. 2273-2289.

[18] M. Iturralde, T. A. Yahiya, A. Wei and A. Beylot, "Resource allocation using shapely value in LTE networks", IEEE International Symposium on Personal Indoor and Mobile Radio Communications Conference, (2011) September, pp. 31-35.

[19] YouTube Traces from the Campus Network, 2008. Available: http://traces.cs.umass.edu/index.php/Network/Network.

[20] M. Zink, K. Suh, Y. Gu and J. Kurose, "Watch Global Cache Local: YouTube Network Traces at a Campus Network - Measurements and Implications", IEEE MMCN, (2008) January.

[21] E. Perahia and R. Stacey, "Next generation wireless LANs: throughput, robustness and reliability in 802.11n", Cambridge University Press, (2008).

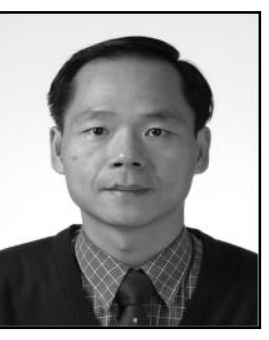

\section{Authors}

Po-Jen Chuang, received the B.S. degree from National Chiao Tung University, Taiwan, R.O.C., in 1978, the M.S. degree in Computer Science from the University of Missouri at Columbia, U.S.A., in 1988, and the Ph.D. degree in Computer Science from the Center for Advanced Computer Studies, University of Southwestern Louisiana, Lafayette, U.S.A. (now the University of Louisiana at Lafayette), in 1992. Since 1992, he has been with the Department of Electrical Engineering, Tamkang University, Taiwan, where he is currently a Professor. He was the department chairman from 1996 to 2000. His main areas of interest include parallel and distributed processing, fault-tolerant computing, computer architecture, mobile computing, network security and cloud computing. 


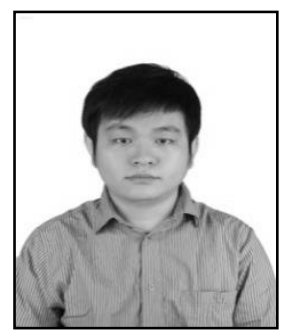

Hang-Li Chen, received his B.S. and M.S. degrees in Electrical Engineering in 2011 and 2014 from Tamkang University, Taiwan. His research interests include distributed processing, content delivery networks and mobile computing. 